\title{
Zonulin as a biomarker and potential therapeutic target in multisystem inflammatory syndrome in children
}

\author{
Tiffany Hensley-McBain ${ }^{1}$ and Jennifer A. Manuzak² \\ 'Clinical Care Options, Reston, Virginia, USA. ${ }^{2}$ Division of Immunology, Tulane National Primate Research Center, Tulane University, Covington, Louisiana, USA.
}

\begin{abstract}
Multisystem inflammatory syndrome in children (MIS-C) occurs during or recently following severe acute respiratory syndrome coronavirus 2 (SARSCoV-2) infection and is characterized by persistent fever, inflammation, and severe illness requiring hospitalization. The majority of patients with MIS-C also present with gastrointestinal (GI) symptoms, including abdominal pain, vomiting, and diarrhea. In this issue of the $J C l$, Yonker, Gilboa, and colleagues identified zonulin as a biomarker of GI permeability in children with MIS-C and present the results of an intriguing proof-of-concept study indicating that zonulin may represent a potential therapeutic target for MIS-C treatment and prevention. Their findings suggest that intestinal mucosal dysfunction and epithelial barrier breakdown may represent a biological mechanism underlying the development of MIS-C in SARS-CoV-2-infected children.
\end{abstract}

Identification of MIS-C in SARS-CoV-2-infected children

Since the first reported cases of severe acute respiratory syndrome coronavirus 2 (SARS-CoV-2), the causative agent of coronavirus disease 2019 (COVID-19) (1), the WHO has reported over 173.5 million confirmed cases of COVID-19 worldwide and more than 3.7 million deaths (2). Throughout the pandemic, the COVID-19 situation for children has differed from that of adults. Indeed, most reports indicate that although children are equally likely to become infected with SARS-CoV-2, the disease is mild for a large majority of them (3). As such, the COVID-19 mortality rate in the United States is 0.34 per 100,000 population and 0.16 per 100,000 for children aged 0-4 and 5-14 years, respectively (4). In stark contrast, the overall COVID-19 mortality rate for adults is 91.5 per 100,000 population, with the highest rate for those aged 85 years and older (1797.8 per 100,000; ref. 5).
In April 2020, reports of children with severe complications that developed days to weeks after SARS-CoV-2 infection or exposure began to emerge (6). In these cases, otherwise healthy children were hospitalized with cardiogenic shock or Kawasaki disease-like symptoms, including persistent fever, abdominal pain, vomiting, diarrhea, skin rash, mucocutaneous lesions, and, in severe cases, hypotension and shock (7). On the basis of these observations, the CDC issued a health advisory including a case definition for multisystem inflammatory syndrome in children (MIS-C) associated with COVID-19 (7). The CDC guidance describes MIS-C as occurring in individuals under the age of 21 years who present with fever, laboratory evidence of inflammation (including an increased erythrocyte sedimentation rate [ESR] as well as elevated levels of C-reactive protein [CRP], D-dimer, IL-6, etc.), and severe illness with multisystem organ involvement and requiring hospitalization. In addition, to meet the case definition,

Related Article: https://doi.org/10.1172/JCI149633

Conflict of interest: The authors have declared that no conflict of interest exists.

patients must demonstrate evidence of current or recent SARS-CoV-2 infection by reverse transcription PCR (RT-PCR), antigen test, or serology test, or exposure to a suspected or confirmed case of COVID-19 within four weeks of symptom onset (7). As of June 2, 2021, the CDC has reported 4018 individuals who met these criteria for MIS-C and 36 MIS-C-related deaths in the United States (8). Multiple immunological mechanisms for MIS-C have been proposed, including an inflammatory response to SARS-CoV-2 superantigen (9), delayed IFN response resulting in the distinct MIS-C cytokine storm (10), and production of pathologic autoantibodies (11). Notably, most children with MIS-C test positive for antibodies against SARSCoV- 2 rather than SARS-CoV- 2 by RT-PCR $(10,12)$, supporting the proposed mechanism of dysregulated immune responses to infection rather than pathology driven directly by the virus. However, an autopsy report of a child who died of cardiac failure related to MIS-C showed evidence of SARS-CoV-2 RNA and viral particles in cardiac tissue, suggesting that a second wave or low-level ongoing viral replication may contribute to fatal outcomes (13).

Gastrointestinal dysfunction as a mechanism underlying MIS-C Interestingly, more than $80 \%$ of patients with MIS-C present with gastrointestinal (GI) symptoms (12), as compared with the $10 \%-15 \%$ of adults with COVID-19 infection who report GI manifestations (14, 15). These differences in the prevalence of GI symptoms, combined with the delay between infection and clinical presentation of MIS-C, suggest that GI pathology in MIS-C may occur via a mechanism distinct from that which causes GI disruption in adults with active COVID-19. Recent reports have suggested that microbial dysbiosis and GI barrier breakdown may drive inflammation and immune activation in severe COVID-19 infection in adults (16, 17); however, little is known about GI dys- 


\section{Healthy children}

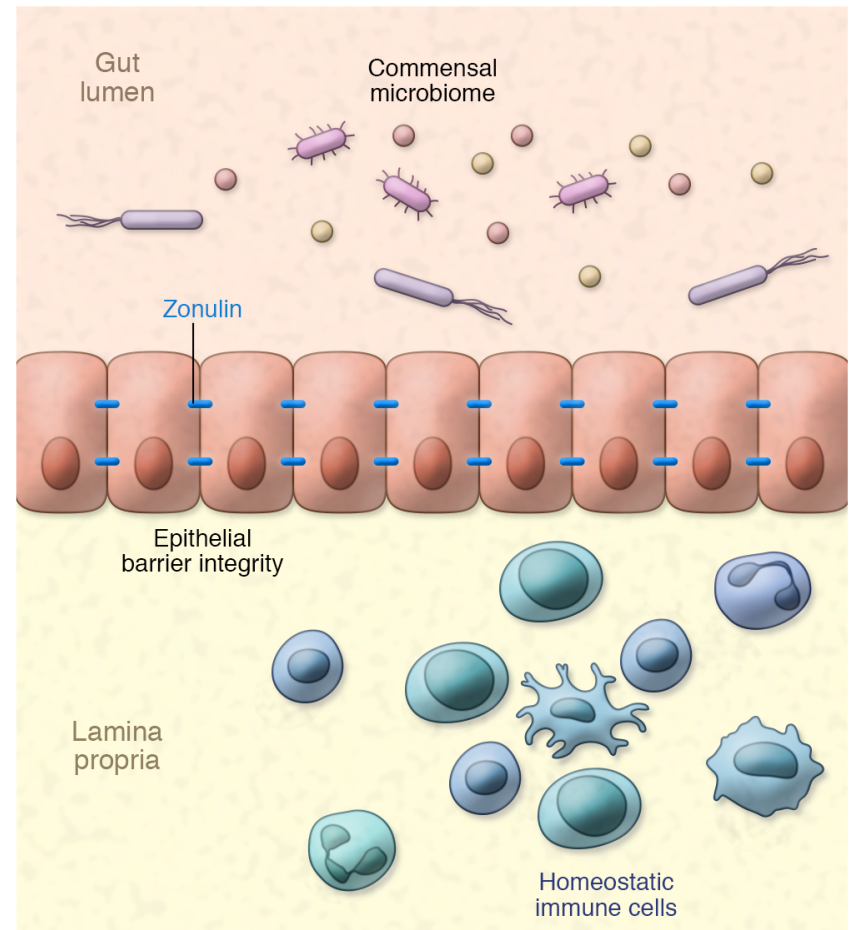

MIS-C

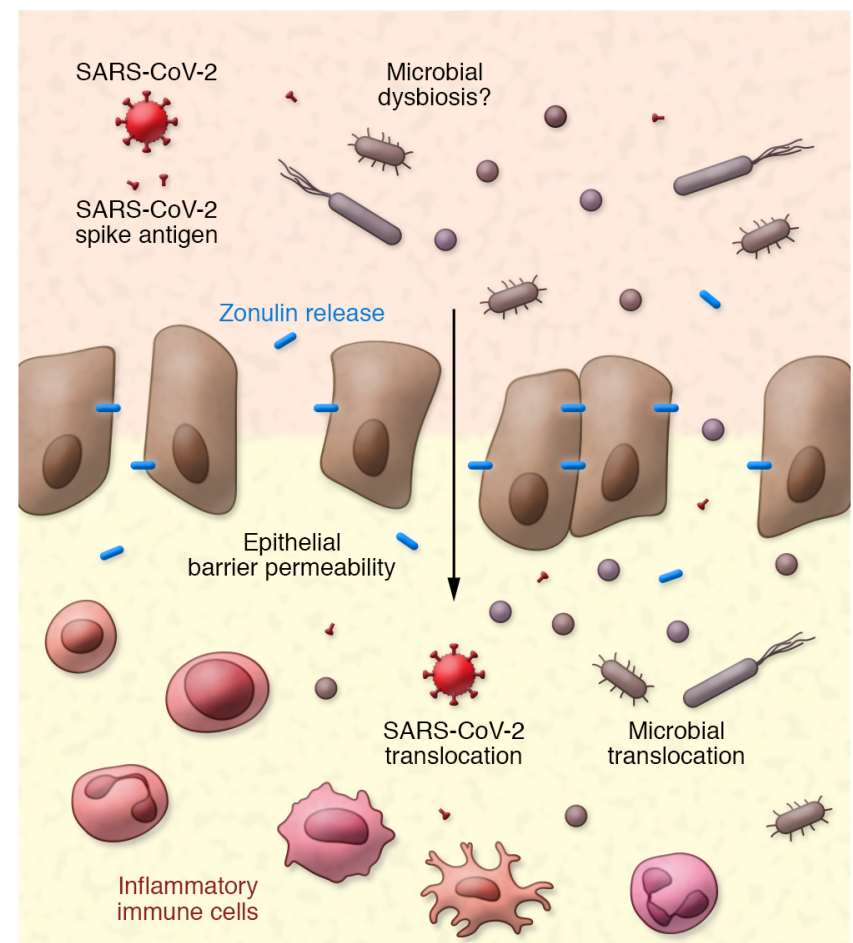

Figure 1. Potential model of zonulin-dependent intestinal permeability in MIS-C. In healthy children, an intact epithelial barrier separates the lumen of the gut and the commensal microbiome from homeostatic immune cells that reside in the lamina propria. In some children with SARS-CoV-2 infection or exposure, zonulin-dependent intestinal barrier breakdown results in elevated plasma zonulin levels and may allow for SARS-CoV-2 and S1 spike antigen, as well as other gut-residing microbes and microbial particles, to translocate into the circulation. Subsequent activation of inflammatory immune cells may contribute to the development of MIS-C.

function in MIS-C. The study by Yonker, Gilboa, and colleagues in this issue of the JCI addresses the potential role of GI disruption in MIS-C by measuring the levels of zonulin, a proposed biomarker of intestinal permeability (18).

Zonulin modulates epithelial barrier integrity by triggering a signaling cascade that results in the phosphorylation and displacement of tight junction proteins (19). Increased GI permeability through a zonulin-dependent mechanism allows paracellular passage of antigenic triggers from the gut lumen into the mucosa and, eventually, the systemic circulation. Zonulin-mediated disruption in the GI tract has been implicated as a pathological mechanism underlying multiple chronic autoimmune and hyperinflammatory diseases (20). Notably, some studies have proposed that zonulin can be viewed as a marker of enterocyte function, suggesting that decreased plasma levels of zonulin could indicate epithelial cell death or dysfunction $(21,22)$. Indeed, lower plasma zonulin concentrations in individ- uals living with HIV who are treated with antiretroviral therapy predicted mortality in one cohort study (21). While it is possible that reduced zonulin levels may represent epithelial cell death or dysfunction, multiple studies in which inhibition of zonulin prevented immune-mediated disease support a role for zonulin in pathological intestinal permeability (Figure 1) (19).

\section{Zonulin-dependent intestinal permeability in MIS-C}

Yonker, Gilboa, and coauthors (18) demonstrated that children with MIS-C had elevated plasma zonulin levels and detectable SARS-CoV-2 viral loads in the stool, suggesting ongoing intestinal viral replication. These findings led the authors to hypothesize that intestinal barrier breakdown could allow for SARS-CoV-2 antigenemia, thus leading to the development of MIS-C. In support of this, the authors demonstrated that children with MIS-C exhibited elevated plasma levels of SARS-CoV-2 spike protein, specifically the S1 component, as compared with SARS-CoV-2-infected children without MIS-C or healthy controls. Finally, the authors conducted an intriguing proof-of-concept experiment in which they treated a child with MIS-C with the zonulin antagonist larazotide (23). Following larazotide treatment, the child showed clinical improvement, including reduced plasma levels of CRP, inflammatory cytokines, and spike antigens. These findings presented by Yonker, Gilboa, and colleagues suggest that therapies focused on rescuing intestinal barrier integrity may be a viable option for the treatment or prevention of SARSCoV-2-associated MIS-C (18).

\section{Concluding thoughts}

Yonker, Gilboa, and coauthors (18) acknowledge that more work remains to be done to conclusively identify the gut as the source of the elevated levels of circulating SARSCoV-2 viral particles that may trigger the development of MIS-C. In particular, studies are needed to assess the ability of SARSCoV-2 antigens to traverse the intestinal 
mucosal epithelial barrier and to verify that zonulin disruption is the major mechanism mediating translocation. Additionally, it will be important to determine how simultaneous translocation of SARS-CoV-2 proteins and additional microbial components may facilitate the risk for MIS-C. Indeed, Yonker, Gilboa, and colleagues (18) demonstrated that plasma levels of the microbial translocation markers lipopolysaccharide-binding protein (LBP) and soluble CD14 (sCD14) were elevated in children with MIS-C as compared with levels in the controls or in children with SARS-CoV-2 without MIS-C. Furthermore, the role of microbial dysbiosis in intestinal barrier permeability and the risk for MIS-C remain unclear. Previous studies have shown that microbial dysbiosis occurs during SARS-CoV-2 infection in adults and identified a potential link between disrupted intestinal microbial profiles and the risk of severe COVID-19 $(24,25)$. Given these findings and the importance of the commensal microbiome in maintaining intestinal immunity and barrier integrity (26), it will be critical to define (a) the impact of SARS-CoV-2 infection on microbial community structure in children and (b) how shifts in microbial profiles due to COVID-19 infection may promote the disruption of mucosal epithelial barriers and enhance the risk for MIS-C. Finally, it is interesting to speculate whether a method of microbial manipulation to restore intestinal microbial communities, such as the use of probiotics or prebiotics or fecal microbial transplantation, could synergize with larazotide to reduce mucosal permeability and limit the risk for and pathology of MIS-C.

Taken together, the importance of identifying viable treatment options to prevent or mitigate MIS-C is underscored by the increasing prevalence of SARS-CoV-2 infections in children and adolescents and the current unavailability of COVID-19 vaccines for children under the age of 12 . The work presented by Yonker, Gilboa, and coauthors (18) provides insight into a potential mechanism by which zonulin-dependent loss of intestinal epithelial barrier integrity precipitates the translocation of SARS-CoV-2 proteins from the gut, thereby inducing the development of MIS-C. The proof-of-concept data demonstrating that larazotide treatment resulted in clinical improvement of a child with MIS-C warrants further study of potential therapeutic strategies focused on restoring epithelial barrier integrity in SARS-CoV-2-infected children. Moreover, these findings may have wider implications, as this approach could be explored in the pediatric setting for a variety of conditions in which loss of mucosal homeostasis is thought to play a role, such as in intestinal bowel diseases or other viral infections, like HIV.

\section{Acknowledgments}

The authors would like to thank Jennifer Dubin for assistance with editing and proofreading this article. JAM is supported in part by NIH grant R21OD031435 and the NIH base grant P51OD011104 to the Tulane National Primate Research Center.

Address correspondence to: Jennifer A. Manuzak, Tulane National Primate Research Center, 18703 Three Rivers Road, Covington, Louisiana 70433, USA. Phone: 985.871.6473; Email: jmanuzak@ tulane.edu.

1. Wu F, et al. A new coronavirus associated with human respiratory disease in China. Nature. 2020;579(7798):265-269.

2. World Health Organization. WHO Coronavirus (COVID-19) Dashboard. https://covid19.who.int/. Updated June 9, 2021. Accessed June 9, 2021.

3. Brodin P. Why is COVID-19 so mild in children? Acta Paediatr. 2020;109(6):1082-1083.

4. Bhopal SS, et al. Children and young people remain at low risk of COVID-19 mortality. Lancet Child Adolesc Health. 2021;5(5):e12-e13.

5. Ahmad FB, et al. Provisional mortality data United States, 2020. MMWR Morb Mortal Wkly Rep. 2021;70(14):519-522.

6. Sancho-Shimizu V, et al. SARS-CoV-2-related MIS-C: a key to the viral and genetic causes of Kawasaki disease? J Exp Med. 2021;218(6):e20210446.

7. Centers for Disease Control and Prevention. Information for Healthcare Providers about Multisystem Inflammatory Syndrome in Children (MIS-C). https://www.cdc.gov/mis-c/hcp/. Updated May 20, 2021. Accessed June 9, 2021.

8. Centers for Disease Control and Prevention. Health Department-Reported Cases of Multisystem Inflammatory Syndrome in Children (MIS-C) in the United States. https://www.cdc. gov/mis-c/cases/index.html. Updated June 4, 2021. Accessed June 9, 2021.

9. Cheng MH, et al. Superantigenic character of an insert unique to SARS-CoV-2 spike supported by skewed TCR repertoire in patients with hyperinflammation. Proc Natl Acad Sci US A. 2020;117(41):25254-25262.
10. Rowley AH. Understanding SARS-CoV-2-related multisystem inflammatory syndrome in children. Nat Rev Immunol. 2020;20(8):453-454.

11. Consiglio CR, et al. The immunology of multisystem inflammatory syndrome in children with COVID-19. Cell. 2020;183(4):968-981.

12. Abrams JY, et al. Multisystem inflammatory syndrome in children associated with severe acute respiratory syndrome Coronavirus 2: a systematic review. J Pediatr. 2020;226:45-54.

13. Dolhnikoff $\mathrm{M}$, et al. SARS-CoV-2 in cardiac tissue of a child with COVID-19-related multisystem inflammatory syndrome. Lancet Child Adolesc Health. 2020;4(10):790-794.

14. Mao R, et al. Manifestations and prognosis of gastrointestinal and liver involvement in patients with COVID-19: a systematic review and meta-analysis. Lancet Gastroenterol Hepatol. 2020;5(7):667-678.

15. Sultan S, et al. AGA institute rapid review of the gastrointestinal and liver manifestations of COVID-19, meta-analysis of international data, and recommendations for the consultative management of patients with COVID-19. Gastroenterology. 2020;159(1):320-334.

16. Giron LB, et al. Plasma markers of disrupted gut permeability in severe COVID-19 patients [preprint]. https://doi.org/10.1101/2020.11.13.2023 1209. Posted on May 15, 2021 medRxiv.

17. Trottein F, Sokol H. Potential causes and consequences of gastrointestinal disorders during a SARS-CoV-2 infection. Cell Rep. 2020;32(3):107915.

18. Yonker LM, et al. Multisystem inflammatory syndrome in children is driven by zonulindependent loss of gut mucosal barrier. J Clin Invest. 2021;131(14):149633.

19. Fasano A. Intestinal permeability and its regulation by zonulin: diagnostic and therapeutic implications. Clin Gastroenterol Hepatol. 2012;10(10):1096-1100.

20. Fasano A. Zonulin and its regulation of intestinal barrier function: the biological door to inflammation, autoimmunity, and cancer. Physiol Rev. 2011;91(1):151-175.

21. Hunt PW, et al. Gut epithelial barrier dysfunction and innate immune activation predict mortality in treated HIV infection. J Infect Dis. 2014;210(8):1228-1238.

22. Hensley-McBain T, et al. Intestinal damage precedes mucosal immune dysfunction in SIV infection. Mucosal Immunol. 2018;11(5):1429-1440.

23. Slifer ZM, et al. Larazotide acetate: a pharmacological peptide approach to tight junction regulation. Am J Physiol Gastrointest Liver Physiol. 2021;320(6):G983-G989.

24. Gou W, et al. Gut microbiota, inflammation and molecular signatures of host response to infection. J Genet Genomics. 2021;157(1):121-141.

25. Kim HS. Do an altered gut microbiota and an associated leaky gut affect COVID-19 severity? mBio. 2021;12(1):e03022-20.

26. Kayama H, Takeda K. Manipulation of epithelial integrity and mucosal immunity by host and microbiota-derived metabolites. Eur J Immunol. 2020;50(7):921-931. 